\title{
Current Status of Clinical Laboratory Reimbursement
}

\section{RICK PANNING}

\section{LEARNING OBJECTIVES}

1. Define the basic difference between retrospective reimbursement and prospective payment as it applies to laboratory services. Describe the impact this has had on the laboratory.

2. Describe how ACA has specifically impacted laboratory reimbursement in at least two ways.

3. Identify current potential threats to laboratory reimbursement.

ABBREVIATIONS: ACA - Accountable Care Act; APC - Ambulatory Patient Classification; CAH Critical Access Hospital; CLFS - Clinical Laboratory Fee Schedule; CMS - Centers for Medicare and Medicaid Services; CPI - Consumer Price Index; DRG - Diagnosis Related Group; FEHB - Federal Employee Health Benefits; IOM - Institute of Medicine; MolDX - Molecular Diagnostics; MS-DRG - Medical Severity Diagnosis Related Group; NLA - National Limitation Amount; OIG - Office of Inspector General; OPPS Outpatient Prospective Payment System; PPS Prospective Payment System; SGR - Sustainable Growth Rate.

INDEX TERMS: Clinical Laboratory Reimbursement, Clinical Laboratory Fee Schedule, Fee-for-Service, Diagnosis Related Group, Prospective Payment System, Sustainable Growth Rate, Competitive Bidding, Laboratory Copayment, OPPS Bundling.

Clin Lab Sci 2014;27(2):119

Rick Panning, MBA, MLS(ASCP) ${ }^{C M}$, Chair, ASCLS Government Affairs Committee' Director of Business Development for Shared Clinical Services, Fairview Health Services, Minneapolis, $M N$

Address for Correspondence: Rick Panning, MBA, MLS(ASCP) ${ }^{C M}, 4894$ Meadow Lane, Shoreview, MN
55126, 651-280-5909, panning4@comcast.net.

\section{Introduction}

In general, reimbursement for laboratory services in this country comes from two third-party payer sources government and private sector. This third-party payment allows patients to access laboratory services from hospitals, physician offices, long-term care and other post acute providers and private laboratories. There are many components to this complex reimbursement system, including how and when tests are covered (coverage decisions), procedure and diagnosis codes (identifying what was actually ordered and performed) and payment methodologies. While levels of reimbursement are intended to reflect the value and the cost of the service being provided, over time the level of payment has been continually reduced to meet financial goals of the government or the healthcare system.

Government payers primarily reflect Medicare on the federal level and Medicaid (Medical Assistance) on the state level. The Medicare program is the most influential reimbursement system since it often impacts state programs and private payer reimbursement policies and payment levels. All public payers and approximately $67 \%$ of private payers use Medicare's payment methodologies as the basis for their own and as tools for negotiating discounts with providers. ${ }^{1}$ In our current fiscal environment, with pressures on the federal budget and concerns about the long term viability of Medicare, we have seen a change in which the Medicare program is beginning to look at private payer reimbursement levels as an opportunity to reduce its financial responsibility for laboratory reimbursement.

The steady reduction in payment levels from Medicare and other payers are resulting in a negative impact on laboratory practice and access. Unfortunately, Congress, rather than calling for the restructure of the federal payment system, continually views the laboratory as a 
source of potential revenue and repeatedly reduces payment to meet federal financial needs. The same can be said for the Medicaid program and private third party payers. There continues to be ongoing discussion of how laboratory reimbursement, when regarded as a commodity, can continue to be reduced or restructured. Concepts, including co-insurance or copayments and competitive bidding remain on the radar as a way to reduce the federal government's share of the responsibility for the reimbursement of laboratory services.

Although healthcare reform, under the 2010 Accountable Care and Patient Protection Act, is intended to make much-needed change in this area, the Medicare statute has historically restricted payment for screening and other preventive technologies and services, unless otherwise specified by Congress.

Currently Medicare reimbursement varies across 56 different fee schedules based on geography. This adds unnecessary bureaucracy, waste and complexity to the federal payment system. There are significant differences in reimbursement for the same tests that are ordered frequently and are often referred to as "routine" tests. Conversely there are many tests on the Clinical Laboratory Fee Schedule (CLFS) for which the current level of reimbursement is not sufficient to cover the costs of performing the tests. Due to the lack of comparable and reliable cost data for laboratory testing, there is an unknown relationship between reimbursement levels and cost. We know that the cost of production varies significantly based on volume and economies of scale, as well as technology and the type of testing performed - acute care hospital, physician office, large automated reference laboratories, etc.

Another deficiency in the current payment models is a lack of transparency in terms of how payment levels are initially established and updated. The current Centers for Medicare and Medicaid Services (CMS) concepts of cross-walk and gap-filling are inconsistently utilized and do not reflect the appropriate analysis of the true cost to perform testing. ${ }^{2,3}$

If the quality, safety and outcomes for patient care are going to be improved and even transformed, one of the primary enablers will be clinical laboratory testing. Maintaining an adequate and dependable workforce, implementing comprehensive and reliable information systems, developing quality systems and implementing state of the art technology will require adequate financial resources and reimbursement methodologies need to reflect this continual change dynamic of the industry. Demonstration of the value of laboratory testing will be imperative if reimbursement systems are to effectively reflect that value.

This article will describe the mechanisms by which the Medicare program, and thus most other payment programs, have reimbursed laboratory services in this country over time. It will also address the current status of laboratory service reimbursement and the potential threats for the near term future.

\section{Once Upon a Time - The Way it Used to Be}

The Medicare program was voted into law in 1965 and eligible beneficiaries were able to enroll in the program in July of 1966. In the beginning, reimbursement for all Medicare services was based on what is called cost-based fee-for-service payment, which was determined retrospectively, after the service was provided. If a beneficiary received a service, either inpatient or outpatient, the provider organization - hospital, physician office, private laboratory - billed their customary charge to Medicare and was generally reimbursed at that rate or at an appropriate cost-based level. Under this system, Medicare made interim payments to hospitals throughout the hospital's fiscal year. At the end of the fiscal year, the hospital filed a cost report and the interim payments were reconciled with "allowable costs" which were defined in regulation and policy. Under this payment system, Medicare's hospital costs increased dramatically; between 1967 and 1983 , costs rose from $\$ 3$ billion to $\$ 37$ billion annually. ${ }^{4}$

This basic concept stayed in place until the early $1980 \mathrm{~s}$ when both inpatient and outpatient reimbursement structures changed dramatically. It is at this point that the mechanisms for payment diverged for services provided during a hospital stay (inpatient) and those provided in an outpatient or clinic setting.

In order to control the rapidly escalating costs of providing Medicare services, in 1982 Congress mandated the creation of a prospective payment system (PPS) to control costs. This had been tried in many 
states successfully. This system is a per-case reimbursement mechanism under which inpatient admission cases are divided into relatively homogeneous categories called diagnosis-related groups (DRGs). In this DRG prospective payment system, Medicare pays hospitals a flat rate per case for inpatient hospital care so that efficient hospitals are rewarded and inefficient hospitals are penalized and have an incentive to become more efficient. In the beginning there were 499 DRG classifications, which increased to 538 over time. In 2007 the structure was altered with the classifications being called Medical Severity DRGs (MS-DRGs) and there are now 745 MS-DRG classifications which allow for severity adjustment, rather than assuming that all patients in a specific DRG category (i.e. congestive heart failure) are the same. For example, DRG 127 was no longer the DRG for principal diagnosis congestive heart failure (CHF). In place of DRG 127 are three new MS-DRGs 291, 292, and 293, depending upon the secondary diagnoses reported along with the principal diagnosis of $\mathrm{CHF}$, thus reflecting a degree of severity of illness. This system recognizes that there may be major complications and comorbidities or a secondary diagnosis, all of which will impact the amount of services required for treatment. ${ }^{4}$

The principle behind the MS-DRG structure is called case mix complexity and refers to an interrelated set of patient attributes that contribute to the cost of care. Those distinct attributes include severity of illness, risk of mortality, prognosis, treatment difficulty, need for intervention and resource intensity.

The payment adjustments to the DRG fall into four categories. ${ }^{4}$ Since labor is the largest cost component in healthcare, the labor component of the MS-DRG payment can be adjusted based on the wage index for the location of the hospital.

There can be a disproportionate share add-on payment if the hospital treats a high percentage of low income patients. For teaching hospitals, an add-on payment can be made which is called an indirect medical education (IME) adjustment based on the ratio of residents to beds or residents to average daily census. Finally there are outlier payments for specific, costly patients.

Under the DRG, and now MS-DRG system for inpatient reimbursement, the incentives changed dramatically in hospitals. It was now important to understand the overall payment that the hospital would have received for a specific patient and be able to provide the necessary care at a cost below that fixed payment. It was during and following the transition to DRGs and MS-DRGs that we saw the increased focus on reducing length-of-stay, the beginning of a focus on process improvement (Lean and 6 Sigma) and the first stages of beginning to view the laboratory and other ancillary and diagnostic departments as cost centers rather than revenue centers.

In terms of prospective reimbursement, other payers governmental and private, have shifted to this method of payment for inpatient reimbursement. Not all of them are similar to DRGs and prospective payment can take many forms, but in all cases the basic principle of paying for inpatient care in a bundled or packaged form is at the core of the payment structure. In addition to a specific payment system based on diagnosis (i.e. MSDRG) this can take the form of a per-day or per stay type of payment.

There is still a small, continually decreasing, percentage of patients that are paid for based on fee-for service. This is due to a few private payers that still have this payment structure and also for those patients who are self-pay. In the latter, hospitals often have programs to reduce the charges or to develop a reasonable payment plan for the patient.

\section{Outpatient reimbursement}

One of the major changes in healthcare, which resulted from the move to prospective payment systems for inpatients, was a significant, steady transition to treating as much as possible in an outpatient or ambulatory setting. Similar to inpatient laboratory reimbursement, the payment methodology under the Medicare program changed dramatically in the early 1980s, specifically in 1984 when the Medicare Clinical Laboratory Fee Schedule (CLFS) was established. Medicare currently pays for outpatient clinical laboratory tests using a prospective payment system (PPS) established in 1984. Payments for over 1,100 tests are set separately in fee schedules for each of 56 geographic jurisdictions, limited by national fee caps called National Limitation Amounts (NLAs). Payments are based on what laboratories charged in 1983, updated periodically for inflation. Laboratories accept Medicare fees as full 
payment. Unlike most other services reimbursed by the Medicare program, there is no beneficiary cost sharing for the laboratory. This means there is no co-insurance or co-pay requiring the beneficiary to bear part of the responsibility for paying for laboratory services. ${ }^{2}$

As one can see, having these fees based on what laboratories charged in 1983 is problematic for laboratories, especially since the updates that were supposed to occur annually have not occurred 12 times since 1990 and in recent years have actually been reduced. The CLFS is intended to be adjusted based on the annual Consumer Price Index (CPI) which would reflect the ongoing increase in the cost of labor, services and supplies. However these increases were frozen or canceled based on the financial needs of the federal government and the Medicare program specifically. ${ }^{5}$

While less than $2 \%$ of all Medicare spending, clinical laboratory testing has been subject to significant freezes in payments and cuts over the last decade. In fact, a hypothetical test that was reimbursed at $\$ 10.00$ in 1984 would be reimbursed at $\$ 8.32$ today in nominal dollars and just $\$ 3.83$ in real or 1984 dollars. Had this $\$ 10$ test been updated for inflation as it was supposed to be under the 1984 law which established the Clinical Laboratory Fee Schedule that same test would be reimbursed at $\$ 21.72$ today.

Most outpatient laboratory testing is reimbursed under the CLFS - hospital outpatients, clinic patients and hospital-based and private reference laboratories. One exception is patients receiving services in a critical access hospital (CAH). These laboratories, in specific hospitals of 50 beds or less located in areas with population densities in the lowest quartile, are reimbursed on a reasonable cost basis, recognizing the reality of having a higher cost structure.

Following Medicare's lead, most state Medicaid/Medical Assistance programs base their individual fee schedules on the Medicare CLFS. Many are lower than the CLSF and are a specific percentage below the Medicare fee schedule. For example, in Minnesota, the Medicaid fee schedule is $7 \%$ below the Medicare fee schedule. Therefore whenever Medicare freezes or lowers the CLFS, the states, which base their reimbursement on the CLFS, make similar adjustments. In the private payer world, some payers base their payments on the Medicare fee schedule, and similar to state Medicaid programs may have general rules which base their fee schedule on specific percentage of the Medicare CLFS, and in extreme examples it can be at a level of $50 \%$ of the CLFS. Other private payers develop their own fee schedule methodology, pay based on a percentage of submitted charges, or bundle into the overall outpatient visit, a direction that Medicare moved to for hospital outpatients on January 1, 2014. ${ }^{5}$

\section{Current threats to laboratory reimbursement}

We as laboratory services practitioners provide the laboratory data that serve as the foundation for the diagnosis and clinical management of conditions like heart disease, pediatric conditions including leukemia, cancer, diabetes, kidney disease, and infectious diseases as well as management of chronic health conditions. Cuts in reimbursement for laboratory services threaten our ability to meet the needs of physicians and patients, not only for seniors but for all Americans. With the level of cuts being sustained, laboratories cannot hire qualified individuals to perform and interpret these tests, integrate new science into medicine and keep the hundreds of hospital, community and regional laboratories across the United States that provide these services open.

While less than two percent of all Medicare spending, clinical laboratory testing has been subject to significant freezes in payments and cuts over the last two decades. Medicare payment amounts for clinical laboratory services have been reduced by about 40 percent in real (inflation adjusted) terms over the past 20 years.

The cost of clinical laboratory services has increased significantly in the last 25 years, while the clinical laboratory fee schedule has been cut severely. Today, clinical laboratories are paid only 75 percent of the 1984 level when adjusted for inflation. This constitutes a real reduction in reimbursement and not just a reduction in the rate of increase that has been experienced by other health care services. The shrinking Medicare clinical diagnostic laboratory reimbursement is not keeping pace with the changes in cost, technology, complexity and delivery of clinical laboratory services over the past two decades. In recent years, the current reimbursement landscape has continually been a target for federal budget cuts and there are additional threats on the immediate horizon. 


\section{Healthcare Reform - Patient Protection and Accountable Care Act}

Clinical laboratories suffered deep cuts as a result of healthcare reform. The law includes a direct and immediate cut to the Part B Clinical Lab Fee Schedule of 1.75 percent each year from 2011 through 2015 . This nine percent cut is the largest cut among all Part B providers. Clinical laboratories also received another cut through the productivity adjustment-one of only a few providers that were subject to an immediate adjustment in 2011-resulting in a potential additional 11 percent cut over ten years. Together, the direct cut and the productivity adjustment result in a cumulative 20 percent cut over ten years. Specifically for 2014, there will be the combination of the ACA cut and the productivity adjustment of $-0.75 \%$ for the year. Laboratories were also subject to a 2 percent cut to the fee schedule as a result of sequestration, which began in January, 2013. Those sequestration cuts were not reduced for Medicare programs in the recent budget agreement by Congress for 2014 .

Another change that occurred on January 1, 2013, as part of healthcare reform, was the imposition of the medical device tax. The Patient Protection and Affordable Care Act levies a 2.3\% excise tax on the sale and import of Class I, II, and III medical devices. This part of the legislation includes instruments, machines, implants, and in vitro reagents. While the House of Representatives has voted to repeal this new tax, the Senate has not yet acted on the repeal, although a majority of the members of the Senate support it. Basically, labs that purchase these devices could be responsible for paying this tax because suppliers, manufactures, and distributors are likely to pass along these added expenses. In essence this becomes an additional laboratory expense at a time when reimbursement is continually under attack. ${ }^{6,7}$

\section{Office of Inspector General (OIG) Report (2013): Comparing Lab Test Payment Rates: Medicare Could Achieve Substantial Savings}

In 2010 Medicare, as the largest payer of clinical laboratory services, paid approximately $\$ 8.2$ billion for lab tests, which accounted for 3 percent of all Medicare Part B payments. The study compared Medicare reimbursement rates to other health care service payers to determine if Medicare was a prudent purchaser of laboratory services. The Office of Inspector General compared Medicare laboratory reimbursement under the CLFS to all 50 of the state Medicaid programs and to three Federal Employees Health Benefits (FEHB) plans that pay for lab tests on a fee-for-service basis. OIG compared to reimbursement rates for 20 high volume laboratory tests for January 1-March 31, 2011.

What they found was that in 2011, Medicare paid between 18 and 30 percent more than other insurers for 20 high-volume and/or high-expenditure lab tests. Based on this analysis the OIG concluded that Medicare could have saved $\$ 910$ million, or 38 percent, on these lab tests if it had paid providers at the lowest established rate in each geographic area. While most state Medicaid programs and two of the three Federal Employee Health Benefit programs use the Medicare CLFS as a basis for establishing their own fee schedules and payment rates, most actually pay less. In addition, many of these programs also factor in competitor information from private payers in the setting of their fees. Furthermore, many of these programs also have coinsurance, co-payments and deductibles to reduce their share of payment. ${ }^{8}$

Based on this study, the OIG recommended to Congress that the Centers for Medicare \& Medicaid Services (CMS) seek legislation that would allow it to establish lower payment rates for lab tests and consider seeking legislation to institute copayments and deductibles for lab tests. Although this has not yet been acted on, members of Congress are very aware of the study and CMS is investigating whether it has the authority to make such a change. ${ }^{8}$

\section{Sustainable Growth Rate (SGR)}

The Medicare Sustainable Growth Rate (SGR) is a method currently used by the CMS to control spending by Medicare on physician services. It was enacted in 1997 as part of the Balanced Budget Act. The intent was to assure that the growth in physician payments did not exceed the capacity of the economy to support it. For many years, most people have agreed that the methodology is no longer valid and they continue to call for dramatic annual decreases in physician reimbursement. Because those changes would not be sustainable or palatable, Congress has passed a temporary fix each year. There now seems to be appetite in Congress for a permanent fix. Unfortunately there is a significant price tag to making the fix and the concern 
of the laboratory industry is that Congress may decide to further decrease laboratory reimbursement in order to pay for the fix. One suggestion would be to extend the temporary annual reduction of the fee schedule under healthcare reform to be extended beyond the intended 5 years. ${ }^{\text {' }}$

\section{Competitive Bidding}

Competitive bidding is another form of reducing reimbursement. For Medicare it is currently in place for durable medical equipment. In this process, all laboratories in a defined geographical area would be required to bid on the Medicare laboratory business with pricing that would be offered for each CPT procedure code. Depending on the specific model, one or more winning bidders would be selected based on lowest pricing and ability to provide access to the patients in the area. "Losing" bidders may either be excluded for participating or would have to be willing to offer services at the pricing level of the winning bidder(s).

Competitive bidding creates incentives for laboratories that may cause them to compromise quality in an effort to reduce costs and secure the bid. When cost becomes the major driving force in the system, quality suffers. True competition requires the continued viability of qualified providers of laboratory services in diverse settings. Under forced fiscal constraints, as a result of winning a contract, quality may not be maintained. Competitive bidding also restricts patient access to quality laboratory services by driving out small, convenient local laboratories. Decreased access will occur especially in rural and other underserved areas where the small laboratories do not have the operating margins or volumes to become effective competitive bidders.

\section{Co-insurance/Laboratory Copay}

Most patient care services provided to Medicare beneficiaries have a component of payment that involves patient responsibility. In these cases the patient pays a specific dollar amount or percentage for a procedure or episode of care and Medicare pays the rest. This reduces Medicare's share of financial responsibility. For laboratory services, Medicare does not require a copayment or coinsurance, a decision, which was made in 1984 when the laboratory fee schedule became effective. Frequently Congress reconsiders implementing a patient responsibility aspect to laboratory reimbursement, as it would significantly reduce payments by the Medicare program. The problem this would cause for the laboratory is that the laboratory organization would need to collect the copayment directly from the patient. With each individual laboratory test usually being relatively low in price, the amount of effort and cost to collect the copayment would exceed the amount collected. For example, on a laboratory test with a reimbursement of $\$ 10$, a $10 \%$ copayment would be $\$ 1.00$ and the lab would lose money on each transaction and have difficulty collecting the payment. It would essentially lower laboratory reimbursement in the process.

Hospital Outpatient Bundling of Laboratory Testing Effective January 1, 2014, another change from a final rule from CMS resulted in an additional impact on laboratory reimbursement. In this case, it applies specifically to hospital outpatient laboratory testing and is essentially bundling most outpatient laboratory services into the primary reason or procedure for the patient's outpatient visit. This was proposed under the Outpatient Prospective Payment System (OPPS). Currently when an outpatient has clinical laboratory testing, those tests are reimbursed under the CLFS. In the new payment methodology, most tests will be bundled into the Ambulatory Patient Classification (APC), an outpatient version of the DRG. Exceptions that will continue to be separately reimbursed include molecular tests and tests unrelated to the primary reason for the visit. Depending on the patient and test mix, the impact for the hospital will vary. There will be a definite decrease in specific laboratory reimbursement, but that may be partially or completely offset by an increase in the APC reimbursement rate. ${ }^{10}$

\section{Molecular Diagnostics Reimbursement (MoPath or MolDx))}

Previously, molecular tests were reimbursed under a system where a number of stacking codes, which reflect the various components of the molecular testing process, were billed as a group and reimbursed as a sum. In 2013, a change was made where each analyte for molecular testing was assigned one unique code, which would be billed instead of multiple stacking codes. The MoPath or MolDX system was implemented by Palmetto, the Medicare carrier in California and has resulted in significantly decreased reimbursement rates 
when compared to previous reimbursement. Palmetto's purpose was to identify tests, determine coverage, and determine reimbursement. The system eliminates documentation review to support the testing and reimbursement decisions by the carrier are considered final. This change dramatically impacts appropriate reimbursement for the fastest growing segment of clinical laboratory testing. ${ }^{11,12} 13$

\section{Summary}

The continued cuts to laboratory reimbursement and threats of further reductions are completely out of sync with the percentage of total healthcare expenses in this country. Clinical laboratories guide critical decisions at a low cost. While laboratory test results inform a majority of medical decisions, expenditures related to laboratory services comprise only $1.6 \%$ of the Medicare budget. ${ }^{14}$ (Figure 1 )

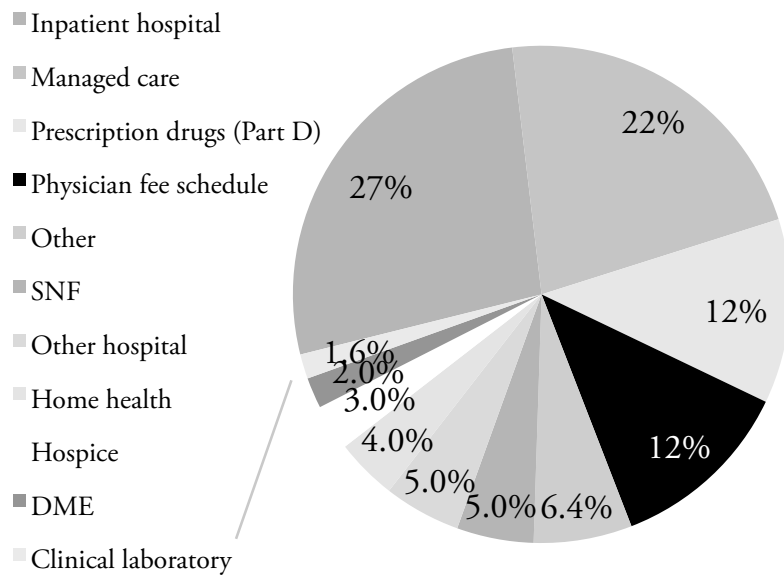

Medicare Payment Advisory Commission (MedPAC).

A Data Book: Health Care Spending and the Medicare

Figure 1. Current State of Reimbursement. Clinical laboratory costs as a percentage of total Medicare expenditures. Source: MedPAC. A Data Book: Halth Care Spending and the Medicare Program. June 2011.

Long-term spending reductions related to deficit reduction initiatives, budget reconciliation and healthcare reform have a material impact on the clinical laboratory industry. These continual cuts have a real impact in our national laboratories, especially small laboratories in hospitals and clinics. Examples of the impacts include the inability to hire personnel, fill positions and working short-staffed, longer hours and overtime with rising supply and labor costs. Continued reimbursement cuts result in budget cuts that threaten the laboratories ability to provide high quality, safe laboratory services. This threatens access to quality patient care in rural and underserved areas.

Clinical laboratories are the only segment of health care that have received real cuts and are reimbursed at rates lower than 1984 when adjusted for inflation. While others may have been frozen or had their increases moderated, no other segment gets paid less today than they did in 1984 .

In 2000, the Institute of Medicine (IOM) did a report of laboratory reimbursement and made 12 recommendations to fix what they called a broken system. Congress and CMS have the opportunity to fix the current Medicare payment system for clinical laboratory services, thus averting the possibility of a crisis in the future. Problems with the outdated payment system could threaten beneficiary access to care and the use of enhanced testing methodologies in the future. If the recommendations were implemented it would improve efficiency of the system and ensure that Medicare beneficiaries continue to have access to high-quality laboratory services. ${ }^{3}$

\section{REFERENCES}

1. Laboratory Medicine: A National Status Report. The Lewin Group. May 2008

2. Clinical Laboratory Services Payment System. Medicare Payment Advisory Committee (MedPAC). October 2013.

3. Medicare Laboratory Payment Policy: Now and in the Future. Institute of Medicine. 2000.

4. Medicare Hospital Prospective Payment System. OEI-09-0000200. Office of Inspector General. August 2001.

5. Aetna Slashes its Laboratory Fee Schedule. Laboratory Economics. April 2013. Access on January 10, 2014 www. laboratoryeconomics.com

6. Summary of the Affordable Care Act. Summary of Coverage Provisions in the Affordable Care Act. Focus on health reform. The Henry Kaiser Family Foundation. April 23, 2013. Accessed November 15, 2013 http://www.kff.org/.

7. Medicare Payment for Clinical Laboratory Tests Likely to be Reduced 2.75\% in 2014. National Intelligence Report. 13(22). December 12, 2013.

8. Comparing Laboratory Test Payment Rates: Medicare Could Achieve Substantial Savings. OE1-07-11-00010. Office of Inspector General. June 2013.

9. Specialist Weigh in on SGR Fix. National Intelligence Report. 14(2). January 23, 2014. 
10. Hospital Outpatient Bundling. National Intelligence Report. 14(3). February 6, 2014.

11. CMS Makes Limited Changes to Final MoPath Rates for 2014; BRCA 1/2 Pricing Remains a Source of Confusion. National Intelligence Report. 13(22). December 22, 2013.

12. Final Medicare NLAs for Most MDx Tests Looks Reasonable.
Laboratory Economics. 2013;8(10).

13. CMS to Publish Final Gap-Fill Rates for MDx Tests. Laboratory Economics. 8(9). September 2013.

14. Medicare Payment Advisory Committee (MedPAC). A Data Book: Health Care Spending and the Medicare Program. June 2011.

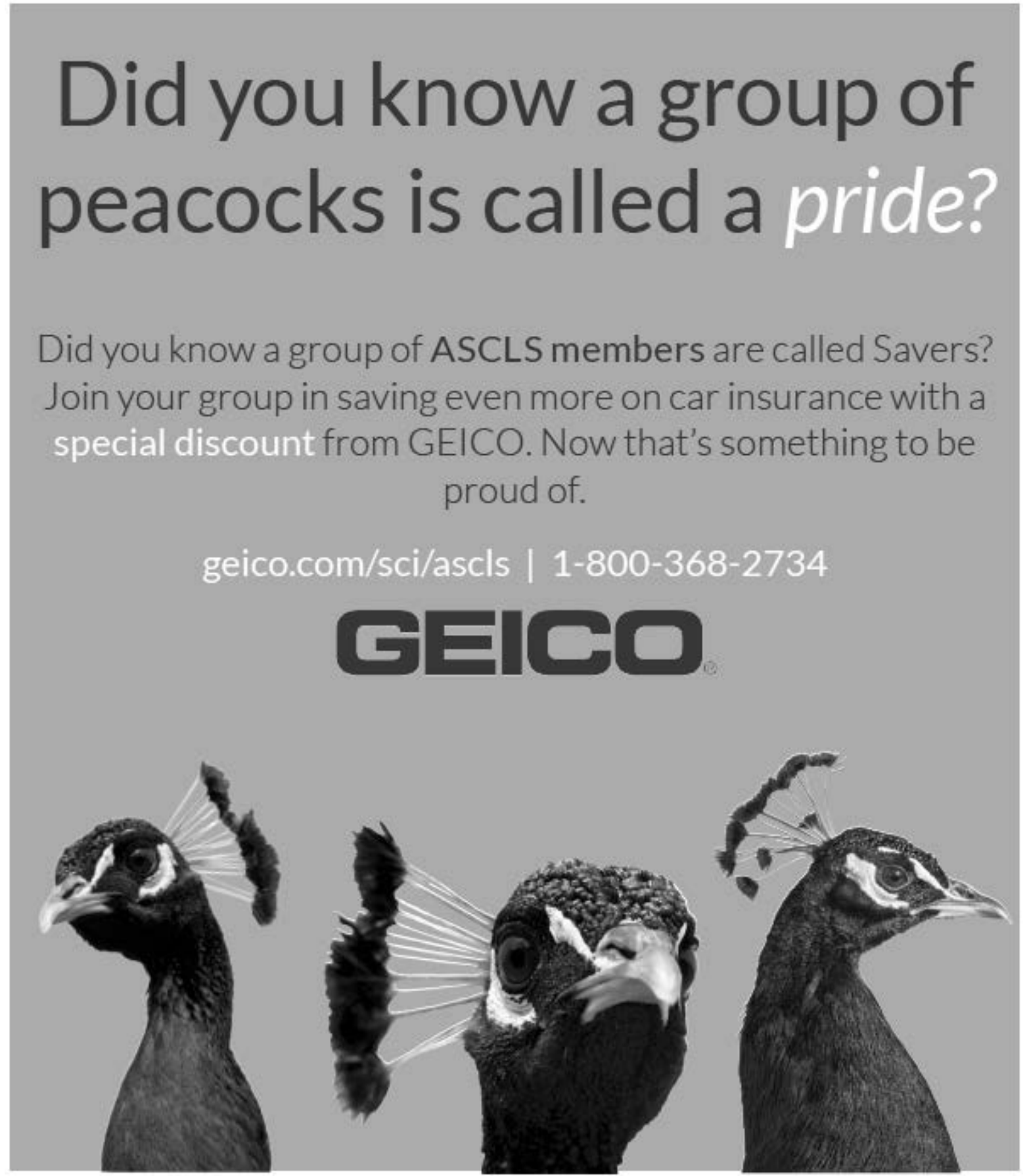

Some discounts, coverages, payment plans and features are not available in all states or all GEICO companies. Discount amount varies in some states. One group discount applicable per policy. Coverage is individual. In New York a premium reduction may be available. GEICO is a registered service mark of Government Employees Insurance Company, Washington, D.C. 20076; a Berkshire Hathaway Inc. subsidiary. @2014 GEICO 